\title{
Mechanical Properties Studied at the Nanoscale Using Scanning Local-Acceleration Microscopy (SLAM)
}

\author{
F. Oulevey, N.A. Burnham, A.J. Kulik, P.-J. Gallo, G. Gremaud and W. Benoit
}

Institut de Génie Atomique, Département de Physique, Ecole Polytechnique Fédérale de Lausanne, 1015 Lausanne, Switzerland

\begin{abstract}
A new technique for studying mechanical properties with a lateral resolution of better than $40 \mathrm{~nm}$ is presented. The Scanning Local-Acceleration Microscope (SLAM) is based on the Atomic Force Microscope (AFM). The principle of the SLAM technique is to vibrate the sample with a small amplitude at a frequency above the tipsample system's highest resonance, forcing the AFM tip to deform locally the sample's surface. This permits one to determine the sample's properties with a dramatically improved signal-to-noise ratio, from both compliant (polymer) and stiff (metal or ceramics) samples. SLAM allows one to measure the "contact stiffness" between tip and sample, which can be related to the elastic modulus of the sample. Present developments explore the possibilities of damping measurements on the same lateral resolution scale, which could lead to a better understanding of nanophased materials and interfaces. One future goal is the passage from qualitative to quantitative measurements. The SLAM instrumentation, the basis of the "stiffness" interpretation and some results from a PVC/PB polymer-blend, from a $\mathrm{NiT}$ /epoxy metal reinforced polymer and from an $\mathrm{Al}_{2} \mathrm{O}_{3} / \mathrm{Al}$ Metal-Matrix Composite are presented. The current status of the damping measurements is described.
\end{abstract}

\section{INTRODUCTION}

The miniaturization of technology and the realization of new materials are increasing the interest in microscopic mechanical properties. For instance, composite materials' stress-transfer properties are mainly controlled by the quality of the interfaces between fibers and matrices [1,2]. To improve both composite fabrication processes and macroscopic properties, it is important to measure local mechanical properties of these interfaces. Such local measurement of a single interface would complement present understanding based on global measurements of materials.

Taking advantage of Atomic Force Microscopy (AFM) [3], where a tip mounted on a very soft spring (cantilever) is in contact with the sample's surface, many ideas have been proposed to measure local mechanical properties [4]. (A more complete set of references can be found in Ref. 5.) The Scanning LocalAcceleration Microscopy (SLAM) principle is to vibrate the sample with a low amplitude at a frequency above the highest system resonance [5]. It has been shown recently [6] to be the optimal setup for measurements of stiff samples. The amplitude of vibration of the tip depends on the "contact stiffness", which can be related to the local elastic modulus. The phase of the vibration depends on energy dissipation in the deformed volume. Hence phase transition studies are possible provided temperature control is achieved.

This paper briefly presents SLAM instrumentation, the tip-sample interaction model and some results from a polyvinylchloride/polybutadiene (PVC/PB) polymer-blend, from a NiTi/epoxy metal reinforced polymer and from an $\mathrm{Al}_{2} \mathrm{O}_{3} / \mathrm{Al}$ Metal-Matrix Composite (MMC).

\section{EXPERIMENT}

SLAM details are described elsewhere [5]. Only its principle is described here (Fig. 1). A transducer is placed beneath the sample and excited with a function generator. The ultrasound is transmitted through the sample, forcing periodic local deformation of the sample's surface underneath the tip. The motion of the AFM tip is detected optically by laser beam deflection from the back side of the cantilever. The detection 


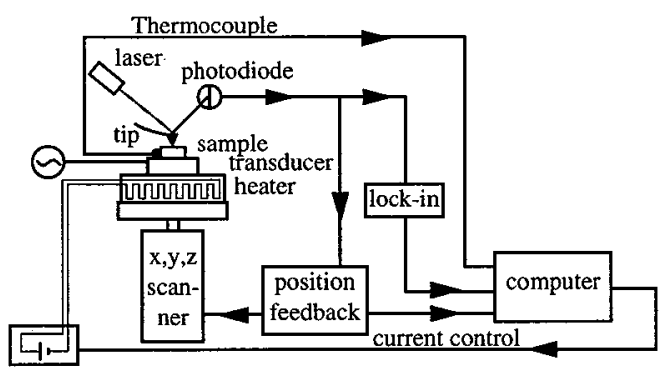

Figure 1: Schematic diagram of SLAM. Ultrasound is generated with a transducer connected to a function generator and placed beneath the sample. The motion of the AFM tip is detected optically. The detection signal is fed to a lock-in amplifier, which extracts amplitude and phase relative to the transducer's motion. The temperature is controlled with a small resistive heater and measured with a thermocouple. The rest of the microscope head, to which the tip, the laser and the photodiode belong, is not represented in this diagram. signal is then fed to a lock-in amplifier, which extracts the tip's amplitude and phase relative to the transducer's motion. The temperature is controlled with a small resistive heater and measured with a thermocouple. For imaging, the output signal of the lock-in is fed into an auxiliary channel of the microscope. An extra computer is used to store spectroscopic data as a function of the temperature at a fixed location.

Working at frequencies above the highest system resonance, the major contribution to forces acting on the tip comes from its acceleration by the sample. Hence Scanning LocalAcceleration Microscope.

\section{TIP-SAMPLE INTERACTION MODEL}

The tip-sample interaction model presented here is a generalization of the model published by Burnham et al. $[5,6]$. It accounts for both elastic and anelastic deformation. Only the major points will be presented here. Exclusively Hertzian contact-mechanics [7] is taken into account. This means that no attractive forces are considered. Imposing a sinusoidal motion to the transducer, the tip's motion will be sinusoidal so long as the nonlinearities of the interaction are weak enough, which implies low vibrational amplitudes. Assuming a single relaxation, the system can be modeled as represented in Fig. 2a and model parameters can be linked to physical parameters through the relations listed in Fig. 2b. It is important to note that the elastic modulus of the sample and the spring constants of the model are connected through an equation including tip modulus and contact radius. As contact radius is not easy to know exactly, SLAM measures "contact stiffness" (i.e., equivalent spring constant in the model at a determined frequency and temperature) and not local elastic modulus. A calibration of the tip is necessary to obtain the value of the elastic modulus.

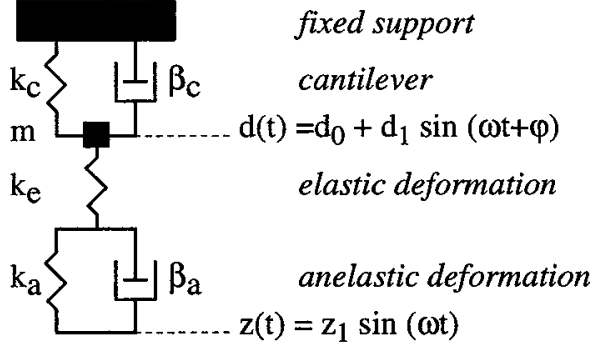

a)

\begin{tabular}{|ll|}
\hline \multicolumn{2}{|c|}{ List of definitions } \\
\hline $\mathrm{a}$ & contact radius \\
$\mathrm{E}_{\mathrm{u}}{ }^{*}$ & reduced unrelaxed elastic modulus \\
$\mathrm{E}_{\mathrm{r}}^{*}$ & reduced relaxed elastic modulus \\
$\mathrm{k}_{\mathrm{c}}$ & cantilever spring constant \\
$\omega_{\mathrm{c}}$ & cantilever free resonance \\
$\beta_{\mathrm{c}}$ & cantilever damping \\
$\tau_{0}$ & relaxation time \\
$\mathrm{E}_{\mathrm{act}} \quad$ activation energy of relaxation \\
$\mathrm{m}=\mathrm{Sqrt}\left[\mathrm{k}_{\mathrm{c}} / \omega_{\mathrm{c}}{ }^{2}\right]:$ cantilever equivalent point-mass \\
$\mathrm{k}_{\mathrm{e}}=2 \mathrm{a} \mathrm{E}^{*} \quad:$ contact-stiffness elastic part \\
$\mathrm{k}_{\mathrm{a}}=2 \mathrm{a}\left(1 / \mathrm{E}_{\mathrm{u}^{*}-1 / \mathrm{E}_{\mathrm{r}}{ }^{*-1}: \text { contact-stiffness anelastic part }}\right.$ \\
$\beta_{\mathrm{a}}=\mathrm{k}_{\mathrm{a}} \tau_{0} \exp \left(\mathrm{E}_{\mathrm{act}} / \mathrm{k}_{\mathrm{B}} \mathrm{T}\right):$ anelastic damping coefficient \\
\hline
\end{tabular}

b)

Figure 2: a) Rheological model of the tip-surface interaction. The AFM cantilever is represented by a spring $k_{c}$, a damper $\beta_{c}$ and a mass $m$. The interaction is represented by a spring $k_{e}$ (elastic deformation) in series with a spring $k_{a}$ and a damper $\beta_{a}$ (anelastic deformation). $z(t)$ is the imposed transducer motion, with amplitude $z_{1}$ and frequency $\omega$. $d(t)$ is the detected tip motion with amplitude $d_{1}$, phase lag $\varphi$ and frequency $\omega$. b) Physical interpretation of the constants introduced in the rheological model.

Using the notation introduced in Fig. 2, the system is described by the equations:

$$
\begin{aligned}
& \frac{d_{1}}{z_{1}}=\sqrt{\frac{k_{a}^{2}+\omega^{2} \beta_{a}^{2}}{\omega^{2} A^{2}(\omega)+B^{2}(\omega)}} \\
& \tan (\varphi)=\omega \frac{\beta_{a} B(\omega)-k_{a} A(\omega)}{\omega^{2} \beta_{a} A(\omega)+k_{a} B(\omega)}
\end{aligned}
$$


Where $d_{1}$ is the tip amplitude, $z_{1}$ the transducer amplitude, $\varphi$ the phase lag,

$\mathrm{A}(\omega)=\left(1+\frac{\mathrm{k}_{\mathrm{a}}}{\mathrm{k}_{\mathrm{e}}}\right) \beta_{\mathrm{c}}+\left(1+\frac{\mathrm{k}_{\mathrm{c}}}{\mathrm{k}_{\mathrm{e}}}\right) \beta_{\mathrm{a}}-\frac{\mathrm{m} \omega^{2}}{\mathrm{k}_{\mathrm{e}}} \beta_{\mathrm{a}}$ and $\mathrm{B}(\omega)=\mathrm{k}_{\mathrm{a}}+\mathrm{k}_{\mathrm{c}}+\frac{\mathrm{k}_{\mathrm{a}} \mathrm{k}_{\mathrm{c}}}{\mathrm{k}_{\mathrm{e}}}-\frac{\beta_{\mathrm{c}} \beta_{\mathrm{a}}}{\mathrm{k}_{\mathrm{e}}} \omega^{2}-\left(1+\frac{\mathrm{k}_{\mathrm{a}}}{\mathrm{k}_{\mathrm{e}}}\right) \mathrm{m} \omega^{2}$.

Both $d_{1} / z_{1}$ (vibration amplitude ratio) and $\varphi$ (phase lag between tip and transducer) can be measured as a function of the temperature and of the position.

The frequency behavior of the tip-sample system in the purely elastic limit $\left(\mathrm{k}_{\mathrm{a}}->\infty\right)$ is studied in detail elsewhere [5]. The major result is that frequencies above the highest system resonance are best suited to distinguish between all kinds of stiffnesses (linear dependence of $\mathrm{d}_{1} / \mathrm{z}_{1}$ on $\mathrm{k}_{\mathrm{e}}$ ) with commercially available cantilevers. Only compliant samples such as polymers can be studied at lower frequencies [8].

For a material going through a phase transition, the anelastic part of the interaction can no longer be neglected. $\varphi$ and $\mathrm{d}_{1} / \mathrm{z}_{1}$ are sensitive to energy dissipation and to elastic modulus changes. Equations 1 and 2 are plotted versus temperature for typical values of PVC mechanical constants around its glass transition temperature $(\mathrm{Tg})$ at a frequency of 700 kHz. $\varphi$ exhibits a peak and $d_{1} / z_{1}$ decreases (Fig. 3) at a temperature which corresponds to the $\alpha$-relaxation at $700 \mathrm{kHz}$. This dependency opens a way towards local mechanical spectroscopy by looking at $\varphi$ or $d_{1} / z_{1}$ as a function of temperature at a fixed location. In addition, imaging $\varphi$ and/or $\mathrm{d}_{1} / \mathrm{z}_{1}$ at several temperatures also allows one to study phase transitions by mapping the dissipative parts of the sample at each temperature ( $\varphi$ images) or changes in dynamic elastic moduli $\left(\mathrm{d}_{1} / \mathrm{z}_{1}\right.$ images). Studying each grain or interphase separately should hence be possible using SLAM, provided a good tefnperature control.

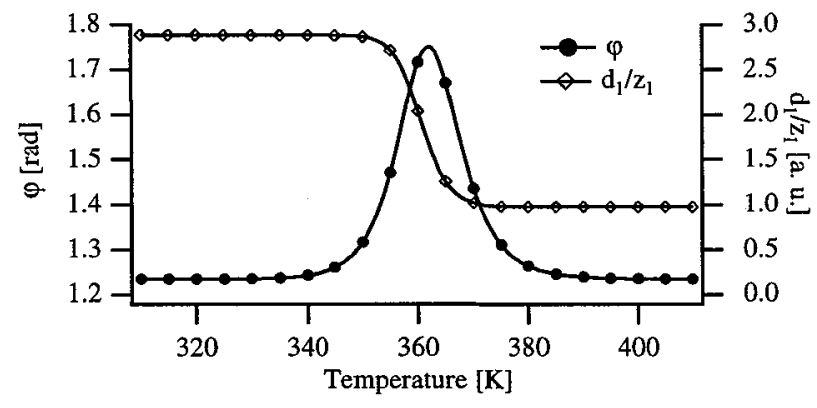

Figure 3: Temperature variation of phase lag $\varphi$ and vibration amplitude ratio $\left(d_{1} / z_{1}\right)$ for typical values of PVC mechanical properties around $\mathrm{Tg}$. The $\alpha$-relaxation causes a change in both $d_{1} / z_{1}$ and $\varphi$.

\section{0}

\section{RESULTS AND DISCUSSION}

AFM normal-force (NF) images are presented together with the SLAM images acquired simultaneously. The NF image represents to first order the topography of the sample's surface. The lighter the pixel, the higher the point. The SLAM image maps $d_{1} / z_{1}$ as a function of the tip's position. They are therefore mapping the local contact stiffness. The lighter the pixel, the stiffer the contact.

\subsection{Elasticity of composite samples}

The SLAM images on both stiff and compliant materials(Fig. 4a-d) show a clear contrast linked to materials properties. The modulus for alumina, aluminum and epoxy are respectively $380 \mathrm{GPa}, 70 \mathrm{GPa}$ and less than $10 \mathrm{GPa}$. The structure of the sample is determined by normal-force AFM or optical microscopy after polishing. $d_{1} / z_{1}$ is greater on stiffer materials. Artifacts due to variation of the contact radius on steep edges can be seen clearly on Fig. 4b. The lateral resolution has been demonstrated elsewhere [5] to be better than $40 \mathrm{~nm}$. As an example of spatial resolution, the dust particles (Fig. 4c-d) are in the tenth of micron range.

\subsection{Phase transitions}

Currently, no reproducible local measurements as a function of temperature on a fixed location ("local mechanical spectroscopy") have been accomplished due to inadequate control of temperature ramps: Images of a sample at temperatures below and above a phase transition allows one to observe the changes in sample's elasticity due to the transition. The sample studied (Fig. 4e-4h) is a polymer blend containing PVC and PB. At room temperature, the PVC is glassy and the PB is rubbery. The PVC glass transition temperature is approximately 60 to $80^{\circ} \mathrm{C}$. The $\alpha$-relaxation associated with this transition should hence be around 100 to $120^{\circ} \mathrm{C}$ at $700 \mathrm{kHz}$ ( $\alpha$-relaxation is thermally activated, the temperature corresponds to glass 
transition temperature around $1 \mathrm{~Hz}$ and shifts with frequency around $7-8^{\circ} \mathrm{C}$ per decade). The NF (topography) image shows clearly that one polymer forms a continuous matrix and the other forms inclusions in it. No clear elasticity contrast can be seen. At higher temperature (around $100 \pm 20^{\circ} \mathrm{C}$ ), the topography changes, due to the difference in thermal expansion coefficients. As the matrix is as stiff as the inclusion at room temperature and much more compliant than the inclusions at high temperature, it is PVC. This is consistent with MT-DSC results obtained on the same kind of sample [9].
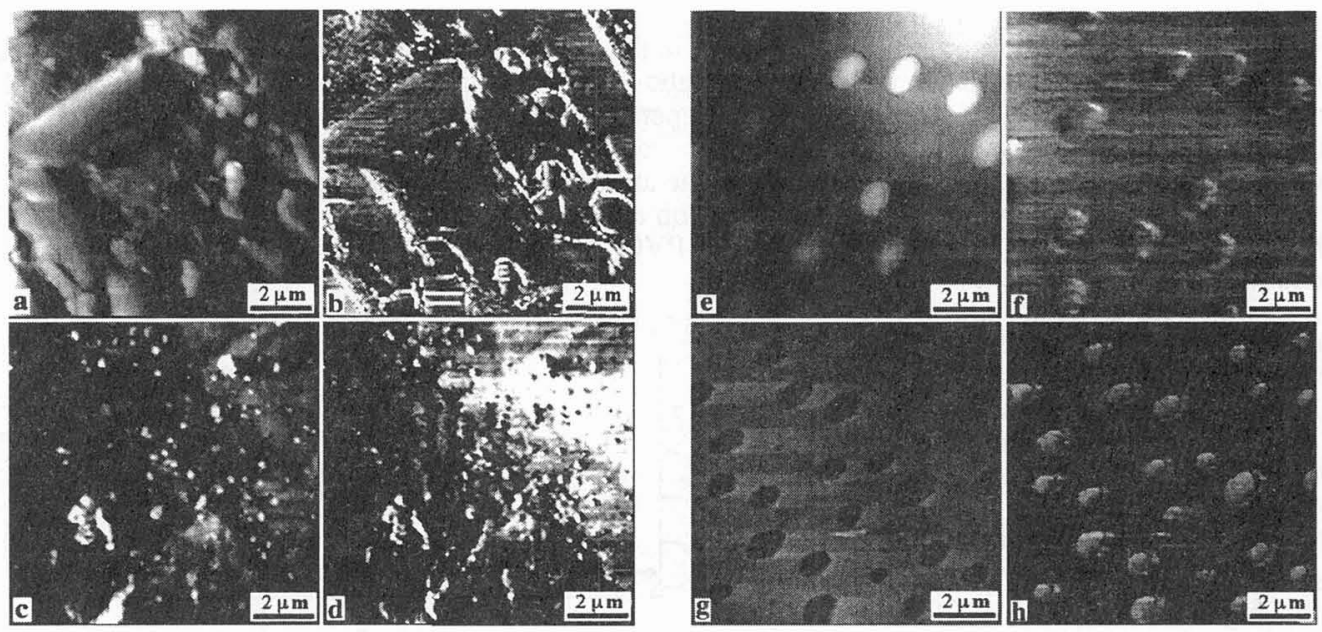

Figure 4: a) NF image ("topography of the sample") of an $\mathrm{Al} 2 \mathrm{O} 3 / \mathrm{Al}$ composite. $\mathrm{Al}_{2} \mathrm{O}_{3}$ platelets emerge from the $\mathrm{Al}$ matrix due to the polishing routine. b) Simultaneous SLAM image: The vibration amplitude of the tip on the alumina platelets is bigger than on the aluminum, as could be expected from the elastic modulus comparison. c) NF image of a NiTi/epoxy composite. Only the interface between the fiber and the matrix can be seen. d) Simultaneous SLAM image: The vibration amplitude of the tip is bigger on the top right, which, from our model, should hence be NiTi. This was confirmed by simultaneous optical microscopy. e-h) Images of a polymer blend at two different temperatures: NF image of a PVC/PB polymer blend below (e) and above (g) PVC glass transition temperature. SLAM image below (f) and above (h) PVC glass transition temperature. As the matrix becomes more compliant (darker) in the SLAM image with increasing temperature, it is composed of PVC and the inclusions are PB. This is consistent with our knowledge of the material [9].

\section{CONCLUSION}

SLAM is suited for mapping elasticity of all kinds of samples. The vibration amplitude ratio $\left(\mathrm{d}_{1} / \mathrm{z}_{1}\right)$ permits one to observe phase transitions with a resolution better than $40 \mathrm{~nm}$. By using the phase lag $(\varphi)$ information, localized mechanical spectroscopy may be possible as well as mapping the dissipative parts of a solid (grain or interphases) at each temperature. Future work will concern the passage from qualitative to quantitative elasticity measurements and the study of phase transitions as a function of temperature.

\section{Acknowledgments}

This work was partially funded by Swiss National Fund for Scientific Research and European Community Human Capital and Mobility Program Project No. CHRX-CT94-0668. Samples were kindly provided by Prof. P. Merle of INSA Lyon, Dr. J.-E. Bidaux from EPFL and Dr. H. Pollock of Lancaster University.

\section{References}

[1] K.K. Chawla, Materials Science and Technology, R.W. Cahn, P. Haasen and E.J. Kramer eds. (VCH, New York, 1993)

[2] S. Goto and M. McLean, Acta Metall. and Mater. 39 (1991) 153-77

[3] G. Binnig, C.F. Quate and C. Gerber, Phys. Rev. Lett. 56 (1986) 930

[4] K. Takata, Jpn. J. Appl. Phys 31 Supplement 31-1 (1992) pp 3-8

[5] N.A. Burnham, A.J. Kulik, G. Gremaud, P.-J. Gallo, F. Oulevey, J. Vac. Sci. Technol. B 14 (1996) 794-99

[6] N.A. Burnham, G. Gremaud, A.J. Kulik, P.-J. Gallo, F. Oulevey, J. Vac. Sci. Technol. B 14 (1996) 1308-12

[7] K.L. Johnson, Contact Mechanics (Cambridge University Press, 1985)

[8] B. Nysten, R. Legras and J.-L. Costa, J. Appl. Phys 78 (1995) 5953-58

[9] A. Hammiche, D.J. Hourston, H.M.Pollock, M. Reading, M. Song, J. Vac. Sci. Technol. B 14 (1996) 1486-91 